\title{
Encefalopatía hepática secundaria a la existencia de un shunt portosistémico tratada satisfactoriamente mediante radiología intervencionista
}

\author{
L. Crespo, J. Graus, F. García-Hoz, R. Bárcena, L. Gil Grande, V. F. Moreira, J. M. Milicua, J. Sánchez y \\ J. Blázquez ${ }^{1}$
}

Servicios de Gastroenterología y ${ }^{\prime}$ Radiología Intervencionista. Hospital Universitario Ramón y Cajal. Madrid

\section{RESUMEN}

La encefalopatía hepática es un estado reversible de alteración en la función cognitiva, que puede ocurrir en pacientes con enfermedad hepática aguda o crónica o shunts porto-sistémicos, en el que puede aparecer cualquiera de los signos neurológicos o psiquiátricos conocidos. Las sustancias nitrogenadas procedentes de la digestión intestinal alcanzan el cerebro sin la depuración que supone su paso por el hígado, debido a las derivaciones porto-sistémicas, y dan lugar a los signos característicos de la encefalopatía hepática. A continuación presentamos dos casos clínicos de pacientes con shunt porto-sistémicos, diagnosticados de encefalopatía hepática crónica recurrente refractaria al tratamiento médico convencional, tratados satisfactoriamente con embolización de dicho shunt mediante técnicas de radiología intervencionista.

Palabras clave: Encefalopatía hepática. Shunt porto-sistémico. Embolización.

\begin{abstract}
Hepatic encephalopathy is a reversible state of altered cognition that may occur in patients with acute or chronic liver disease or porto-systemic shunt, and in which known neurological or psychiatric signs may develop. Nitrogenated substances from intestinal digestion reach the brain without being cleared by their passage through the liver due to the presence of porto-systemic shunt. We report two cases of patients with porto-systemic shunt diagnosed with recurrent chronic hepatic encephalopathy refractory to conventional medical treatment. They were satisfactorily treated with shunt embolization using interventionist radiology techniques.
\end{abstract}

Key words: Hepatic encephalopathy. Porto-systemic shunt. Embolization.

Crespo L, Graus J, García-Hoz F, Bárcena R, Gil Grande L, Moreira VF, Milicua JM, Sánchez J, Blázquez J. Encefalopatía hepática secundaria a la existencia de un shunt portosistémico tratada satisfactoriamente mediante radiología intervencionista. Rev Esp Enferm Dig 2007; 99: 667-670.

\section{INTRODUCCIÓN}

Los pacientes con hepatopatía crónica e hipertensión portal con frecuencia presentan shunts porto-sistémicos espontáneos. Aunque ayudan a reducir la presión portal, la existencia de estos shunts aumenta las sustancias tóxicas circulantes y favorece el desarrollo de encefalopatía hepática. El manejo de este tipo de pacientes debe ser

Recibido: 26-07-07.

Aceptado: 06-08-07.

Correspondencia: Laura Crespo Pérez. Departamento de Gastroenterología. Hospital Universitario Ramón y Cajal. Ctra. de Colmenar, km 9.100. 28034 Madrid. Fax: $9133687 \quad 85$. e-mail: lcreper@yahoo.es I jgraus@ya.com muy cuidadoso ya que como consecuencia directa de la eliminación del shunt se producirá inevitablemente un aumento en la presión portal (1). Los tipos de shunts más frecuentes son el espleno-renal y el gastro-renal (2).

Presentamos dos casos clínicos de encefalopatía hepática crónica recurrente secundaria a la existencia de un shunt porto-sistémico, tratada sastifactoriamente mediante técnicas de radiología intervencionista.

\section{CASO 1}

Mujer de 74 años cirrótica VHC asociada a hemocromatosis estadio B8 de Child con múltiples ingresos con el diagnóstico de encefalopatía hepática severa recurrente (17 ingresos en 20 meses). La ecografía abdominal convencional objetivó un parénquima hepático heterogéneo sin lesiones 
ocupantes de espacio compatible con hepatopatía crónica, una vena porta permeable de $13 \mathrm{~mm}$ y un bazo homogéneo discretamente aumentado de tamaño $(13 \mathrm{~cm})$. Por otro lado, el estudio doppler desmostró la inversión del flujo en las venas porta y esplénica, sin visualizarse un claro shunt portosistémico. Se realizó entonces un angioTC que reveló la existencia de una marcada circulación colateral y un shunt espleno-renal asociado (Fig. 1). La paciente mantuvo cifras de amonio persistentemente elevadas. Mediante radiología intervencionista se procedió a la embolización parcial del shunt con microcoils consiguiéndose un notable enlentecimiento del flujo sanguíneo a través del shunt (Fig. 1). La ecografía abdominal postembolización demostró un flujo portal hepatópeto con persistencia de flujo hepatófugo en la vena esplénica y disminución de la circulación colateral periesplénica.

Tras la embolización la paciente experimentó una mejoría clínica inmediata y las cifras de amonio se normalizaron (Fig. 2). En los 18 meses siguientes a la embolización del shunt ha precisado ingreso hospitalario en tres ocasiones, en relación con descompensación hidrópica leve, síndrome hepatorrenal y encefalopatía hepática grado I. Se realizó una endoscopia oral post-embolización en la que no se objetivaron varices esófago-gástricas.

\section{CASO 2}

Varón de 49 años cirrótico enólico asociado a infección por VHC estadio B7 de Child que ingresa por alteración del nivel de conciencia. Las cifras de amonio durante el ingreso se mantuvieron persistentemente elevadas. Se realizó una ecografía abdominal que demostró a nivel peripancreático un vaso colateral muy dilatado y tortuoso, un parénquima hepático heterogéneo sin lesiones ocupantes de espacio y una esplenomegalia homogénea de $15 \mathrm{~cm}$. Posteriormente se realizó un angioTC que comprobó la existencia de abundante circulación colateral asociada a una derivación portosistémica espleno-renal (Fig. 3).

Mediante técnicas de radiología intervencionista se procedió a la embolización parcial del shunt con microcoils (Fig. 3). La ecografía abdominal postembolización comprobó la desaparición del shunt espleno-renal. Tras la embolización el paciente presentó una rápida mejoría clínica (desaparición de la sonmolencia) y analítica (normalización de las cifras de amonio) (Fig. 2). No ha desarrollado varices gastro-esofágicas ni ascitis. No ha vuelto a precisar ingreso hospitalario durante los 10 meses de seguimiento.

\section{DISCUSIÓN}

Los trastornos metabólicos que acontecen en un paciente cirrótico en estadio terminal favorecen el desarrollo de encefalopatía hepática. Sin embargo, un paciente con shunt porto-sistémico puede desarrollar encefalopatía hepática aún con una función hepática preservada $(1,2)$. Hasta un $46 \%$ de los pacientes cirróticos con marcada circulación colateral desarrollarán encefalopatía hepática. Tradicionalmente, la encefalopatía hepática se ha tratado médicamente y, sólo en casos excepcionales, se ha recurrido a procedimientos invasivos. El tratamiento médico básico consiste en la eliminación de los factores precipitantes, reduciendo fundamentalmente la carga de productos nitrogenados del intestino mediante la administración de disacáridos no absorbibles y/o antibióticos y una dieta pobre en proteínas (3).

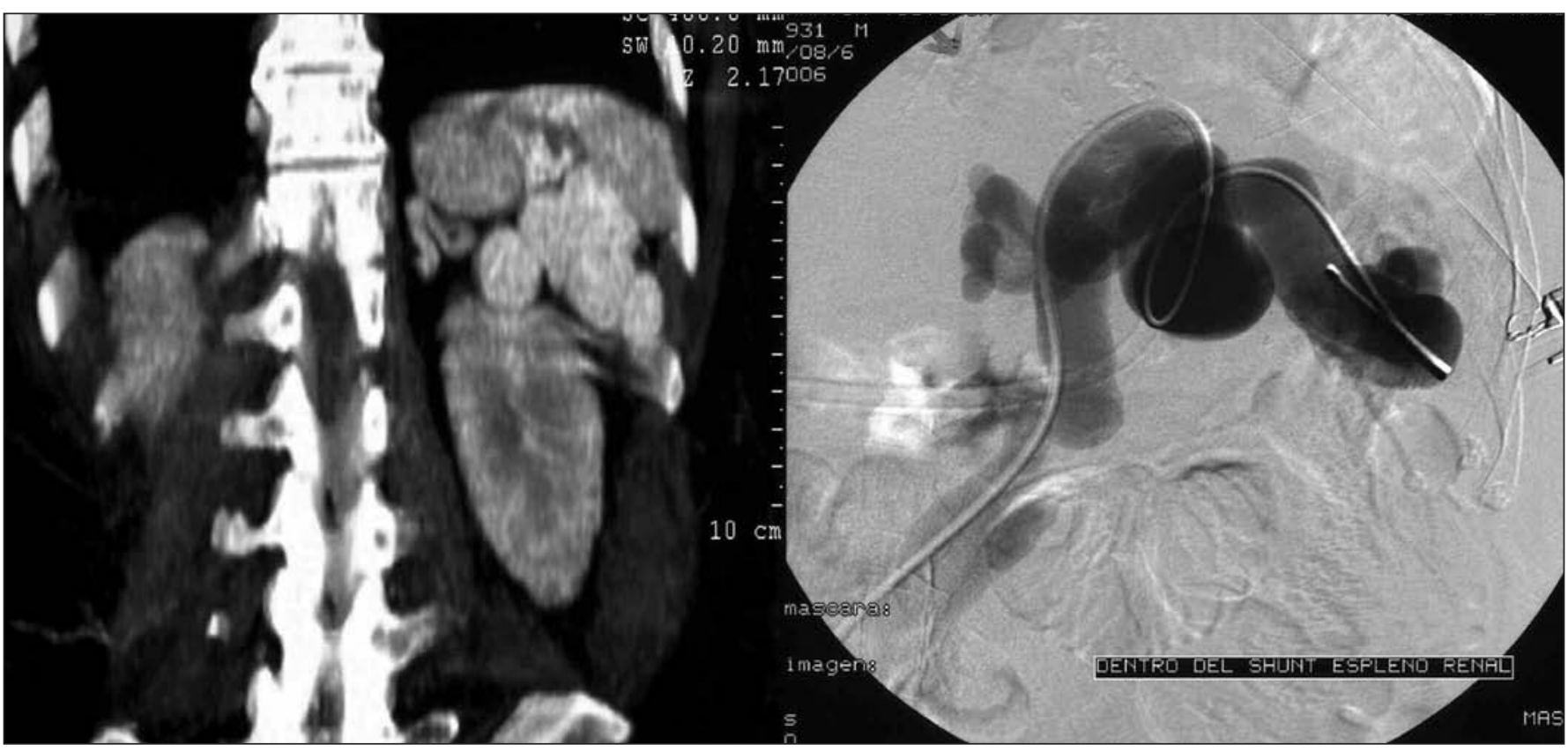

Fig. 1. (Caso 1) A la izquierda, corte coronal de TC en el que se observa una importante circulación colateral. A la derecha, embolización parcial del shunt con micro-coils. 


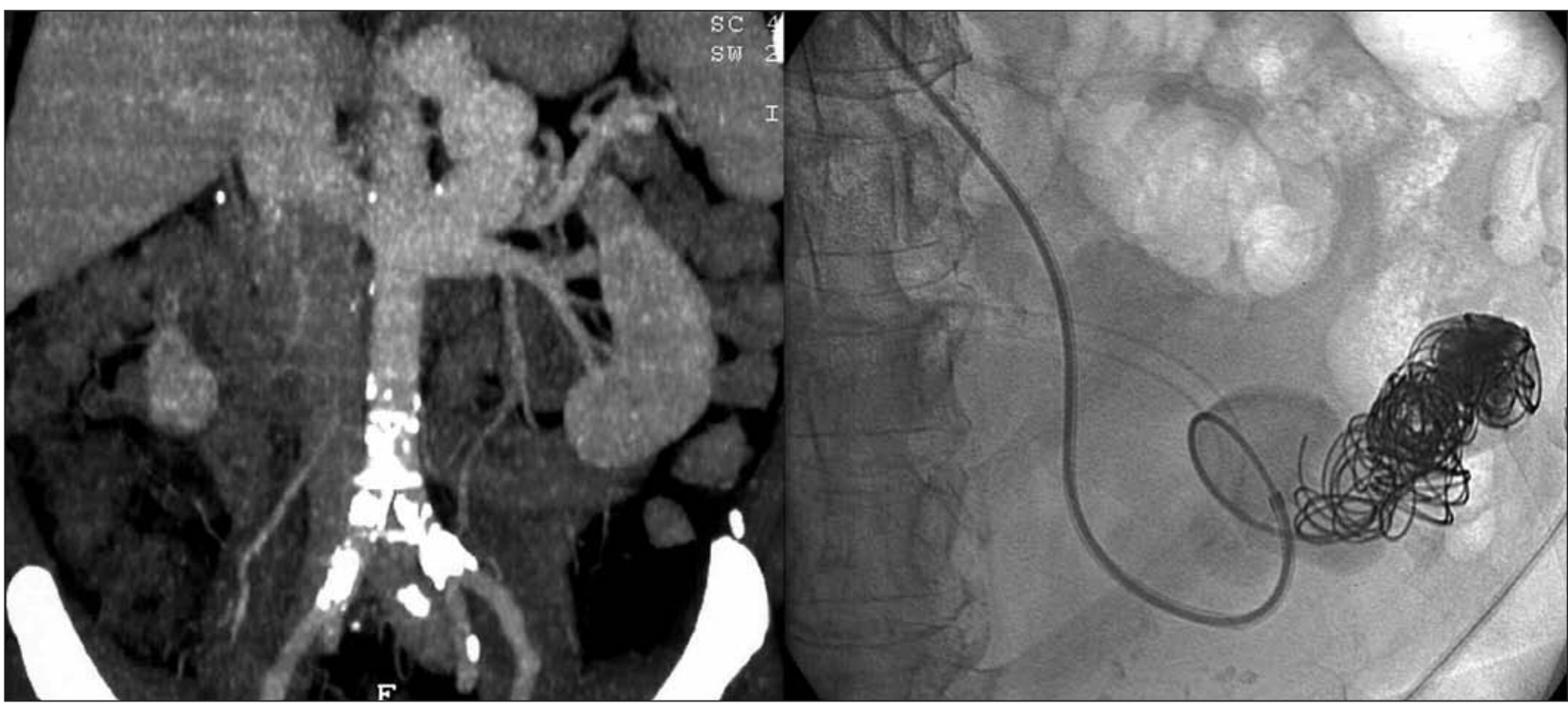

Fig. 2. (Caso 2) A la izquierda, corte coronal de TC en el que se aprecia una marcada circulación colateral. A la derecha, embolización del shunt espleno-renal con micro-coils.

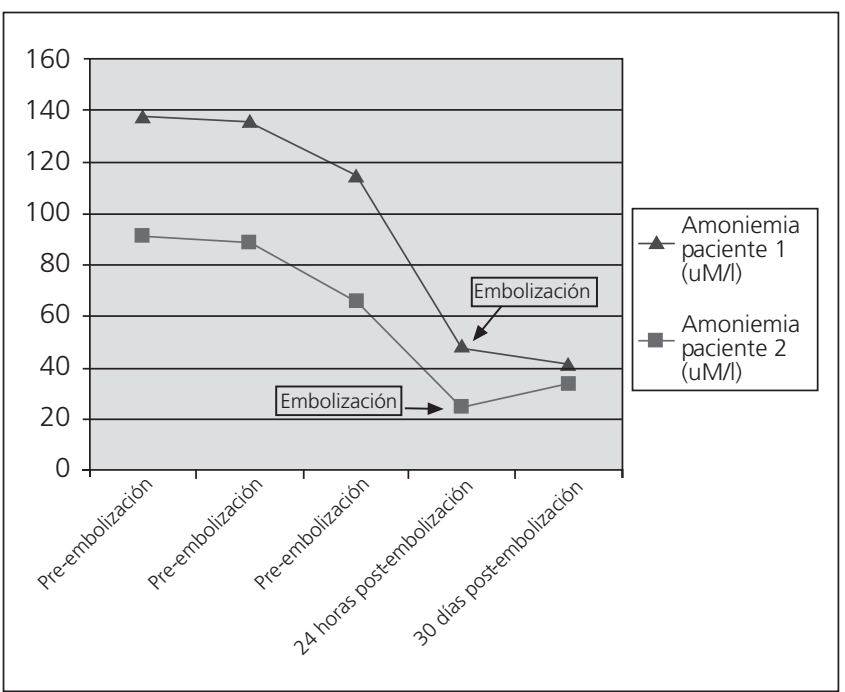

Fig. 3. Evolución de las cifras de amonio tras la embolización del shunt porto-sistémico mediante técnicas de radiología intervencionista. Se observa un claro descenso de la amoniemia tras la embolización, lo que se correlacionó con una importante mejoría clínica en ambos pacientes.

Los shunts porto-sistémicos con frecuencia son causa de encefalopatía hepática recurrente en enfermos cirróticos por lo que se deben tener siempre en cuenta en el diagnóstico diferencial de la encefalopatía hepática recurrente de causa no filiada. En estos casos es frecuente que el tratamiento médico conlleve únicamente mejorías transitorias de la encefalopatía con múltiples recidivas. En estas situaciones, la embolización de los shunts porto-sistémicos consigue una mejoría notable en el nivel de conciencia y un descenso significativo de los niveles de amonio, así como la reinversión del flujo portal (1).
En el 1982 se realizó la primera corrección quirúrgica de un shunt porto-sistémico congénito. Dos años más tarde se realizó la primera oclusión de un shunt mediante técnicas de radiología intervencionista. Kimura y cols. estudiaron 460 shunts porto-sistémicos en pacientes con hipertensión portal. Un 14\% (48 pacientes) tenían shunts gastro-renales izquierdos, $5 \%$ (23 pacientes) shunts gastro-renales posteriores, 6\% (28 pacientes) shunts gastrorenales cortos, $7 \%$ (32 pacientes) shunts espleno-renales, $2 \%$ shunts mesentéricos inferiores, $1,3 \%$ shunts mesentéricos superiores y $0,4 \%$ shunts pancreático-duodenales (4). Takashi y cols. describieron un $15,7 \%$ de shunts esplenorenales y un $5,6 \%$ de shunts gastro-renales en pacientes con hipertensión portal y marcada circulación colateral (5).

Existen algunos casos de encefalopatía hepática con cifras de amonio muy elevadas en pacientes no cirróticos sin hipertensión portal debida a la existencia de shunts portosistémicos, congénitos o adquiridos, aunque su etiología exacta es desconocida y su frecuencia parece estar en aumento quizá debido a la mejoría en las técnicas de imagen $(6,7)$. Algunos de estos casos han podido ser diagnosticados erróneamente de trastornos psiquiátricos (demencia, depresión u otros) (8). Se debe sospechar una encefalopatía hepática secundaria a la existencia de un shunt porto-sistémico cuando las manifestaciones neurológicas sugieran fehacientemente encefalopatía hepática aunque los síntomas objetivos y subjetivos o los valores de laboratorio no sean lo suficientemente indicativos de cirrosis hepática. Por tanto, aún en pacientes sin cirrosis y con función hepática preservada, puede desarrollarse una encefalopatía hepática crónica de difícil tratamiento si no se piensa en la eventual presencia de un shunt porto-sistémico (6).

Las técnicas quirúrgicas son efectivas para controlar la encefalopatía hepática crónica secundaria a la existencia 
de un shunt porto-sistémico, si bien se asocian con altas tasas de mortalidad. Por este motivo en la actualidad se emplean de forma preferente técnicas de radiología intervencionista y prácticamente no se realizan intervenciones quirúrgicas con este fin. Si bien no existe consenso, en general se acepta que la embolización de un shunt portosistémico debe ser realizada cuando el flujo sanguíneo a través del mismo tiene una cuantía importante (flujo invertido), para lo que resulta fundamental disponer de un estudio ecográfico doppler previo a la embolización. Fundamentalmente se utilizan técnicas basadas en la embolización con micro-coils metálicos, si bien puede recurrirse también a la oclusión del shunt mediante el empleo de balones y la inyección de sustancias esclerosantes como el etanol o el oleato de etanolamina. En algunas ocasiones pueden combinarse las dos técnicas anteriores, inyectando primero sustancias esclerosantes y posteriormente introduciendo coils metálicos en el interior del shunt para conseguir una oclusión total del mismo (8).

El cierre de un shunt porto-sistémico puede facilitar el desarrollo de ascitis y la formación de varices esófagogástricas, por lo que estos enfermos deben ser seguidos estrechamente después de este procedimiento. Otras complicaciones más leves descritas en la literatura son el desarrollo de fiebre en las 24-48 horas posteriores a la embolización o la aparición de derrame pleural bilateral de escasa cuantía. De todos modos el efecto adverso más temido es el desarrollo de varices esofágicas o gástricas, que en algunos casos puede incluso presentarse inicialmente en forma de hemorragia masiva (9). Por este motivo algunos autores proponen realizar una embolización parcial esplénica simultáneamente a la embolización del shunt porto-sistémico o en el postoperatorio inmediato. Este tratamiento adicional consigue que el aumento en la presión venosa portal no sea tan elevado como cuando se emboliza el shunt de forma aislada $(10,11)$. Dado el estado general de los enfermos descritos en nuestra comunicación se optó por un seguimiento endoscópico estrecho y no se realizó una embolización esplénica. Ninguno de los dos pacientes descritos desarrolló varices esófagogástricas durante el periodo de seguimiento.

Un estudio reciente realizó un seguimiento a largo plazo ( 5 años) de 25 enfermos con encefalopatía crónica recurrente secundaria a la existencia de un shunt porto-sistémico. Los enfermos fueron divididos en dos grupos: a 14 pacientes se les embolizó el shunt mediante técnicas de radiología intervencionista y a continuación se les realizó una embolización parcial esplénica y a 11 pacientes se les realizó sólo una embolización del shunt porto-sistémico. Los valores de amonio y el grado de encefalopatía hepática en el grupo de pacientes que fueron sometidos además a una embolización esplénica fueron significativamente menores a los 6 meses, 9 meses, 1 año y 2 años tras el procedimiento; por lo que los autores concluyen que la terapia combinada beneficia a los pacientes con encefalopatía crónica recurrente secundaria a la existencia de un shunt porto-sistémico (12). Por otro lado, la em- bolización esplénica podría mejorar la capacidad de síntesis proteíca del hígado y aumentar así las cifras séricas de albúmina, y además reducir el riesgo de hemorragia al mejorar la trombopenia (13).

En conclusión, la encefalopatía hepática crónica recurrente secundaria a la existencia de un shunt porto-sistémico puede presentarse en enfermos tanto cirróticos como no cirróticos. En general este tipo de encefalopatía hepática responde mal al tratamiento médico convencional y actualmente, el tratamiento de elección en los casos que no se controlen satisfactoriamente con tratamiento médico, debería ser la embolización del shunt mediante técnicas mínimamente invasivas basadas en la radiología intervencionista. Dado el elevado riesgo de desarrollo de varices esófago-gástricas tras este procedimiento, parece recomendable asociar una embolización esplénica parcial.

\section{BIBLIOGRAFÍA}

1. Armando Zamora C, Sugimoto K, Tsurusaki M, Yamaguchi M, Izaki $\mathrm{K}$, Taniguchi T, et al. Portosplenic blood flow separation in a patient with portosystemic encephalopathy and a spontaneous splenorenal shunt. J Vasc Interv Radiol 2004; 15: 875-9.

2. Takayama Y, Moriura S, Nagata J, Akutagawa A, Hirano A, Ishiguro $S$, et al. Embolization of the left portal vein to inferior vena cava shunts for chronic recurrent hepatic encephalopathy via the mesenteric vein. J Gastroenterol Hepatol 2001; 16: 1425-8.

3. Carrión JA, Bellot P, Colmenero J, García Pagán JC. Large espontaneous splenorenal shunt as a cause of chronic hepatic encephalopathy. J Hepatol 2004; 40: 868.

4. Kimura K, Ohto M, Matsutani S, Furuse J, Hoshino K, Okuda K. Relative frequencies of porto-systemic pathways and renal shunts formation through the "posterior" gastric vein, Portographic study of 460 patients. Hepatology 1990; 12: 725-8.

5. Takashi M, Takayasu K, Koen H, Musha H, Kotohda K, Okuda K. Extrahepatic portal-systemic shunt and its clinical significance in portal hypertension. Acta Hepatol Jpn 1980; 20: 857-65.

6. Watanabe A. Portal-systemic encephalopathy in non-cirrhotic patients: Classification of clinical types, diagnosis and treatment. J Gastroenterol Hepatol 2000; 15: 969-79.

7. Pocha C, Maliakkal B. Spontaneous intrahepatic portal systemic venous shunt in the adult: A case report and review of the literature. Dig Dis Sci 2004; 49: 1201-6.

8. Taniai N, Onda M, Tajiri T. Combined endoscopic and radiologic intervention to treat esophageal varices. Hepatogastroenterology 2002; 49: $984-8$.

9. Shah SR, Pramesh CS, Deshmukh HL, Mathur SK. Splenic artery embolization for variceal hemorrhage following blocked distal splenorenal shunt. Hepatogastroenterology 2003; 50: 1167-8.

10. Mezawa S, Homma H, Akiyama T, Katsuki S, Murakami K, Hirata $\mathrm{K}$, et al. Selective embolization of the splenic vein in patients with hepatic encephalopathy and splenorenal shunt. J Vasc Interv Radiol 2004; 15: 1475-81.

11. Kato K, Kondo S, Hirano S, Omi M, Ambo Y, Okushiba S, et al. Surgical closure of the gastrorenal shunt with distal splenorenal shunt operation for portosystemic encephalopathy. Hepatogastroenterology 2001; 48: 840-1.

12. Yoshida H, Mamada Y, Taniai N, Yamamoto K, Kaneko M, Kawano, et al. Long-term results of partial splenic artery embolization as supplemental treatment for portal-systemic encephalopathy. Am J Gastroenterol 2005; 100: 43-7.

13. Tajiri T, Onda M, Yoshida H. Long-term haematological and biochemical effects of partial splenic embolization in hepatic cirrhosis. Hepatogastroenterology 2002; 49: 1445-8. 